\title{
Monocyte Reconstitution and Gut Microbiota Composition after Hematopoietic Stem Cell Transplantation
}

\author{
Sejal Morjaria ${ }^{1,7, *},\left(\mathbb{D}\right.$, Allen W. Zhang ${ }^{2}$, Sohn $\mathrm{Kim}^{3}$, Jonathan U. Peled ${ }^{6,7}$, Simone Becattini ${ }^{3}$, Eric R. Littmann ${ }^{3}$, \\ Eric. G. Pamer ${ }^{1,3,4,7}$, Michael C. Abt ${ }^{5, *},+\mathbb{D}$, Miguel-Angel Perales ${ }^{6,7, \dagger}$ \\ ${ }^{1}$ Infectious Disease, Department of Medicine, Memorial Sloan Kettering Cancer Center, New York, NY, USA \\ ${ }^{2}$ Department of Epidemiology and Biostatistics, Memorial Sloan Kettering Cancer Center, New York, NY, USA \\ ${ }^{3}$ Immunology Program and Infectious Disease Service, Memorial Sloan-Kettering Cancer Center, New York, NY, USA \\ ${ }^{4}$ Lucielle Castori Center for Microbes, Inflammation and Cancer, Sloan Kettering Institute, New York, NY, USA \\ ${ }^{5}$ Department of Microbiology, Perelman School of Medicine, University of Pennsylvania, Philadelphia, PA 19104, USA \\ ${ }^{6}$ Adult Bone Marrow Transplantation Service, Department of Medicine, Memorial Sloan Kettering Cancer Center, New York, NY, USA \\ ${ }^{7}$ Department of Medicine, Weill Cornell Medical College, New York, NY, USA
}

\section{ARTICLE INFO}

Article History

Received 07 September 2020

Accepted 21 October 2020

Keywords

Immune reconstitution

monocytes

microbiome

bone marrow transplantation

\begin{abstract}
Background: Monocytes are an essential cellular component of the innate immune system that support the host's effectiveness to combat a range of infectious pathogens. Hemopoietic cell transplantation (HCT) results in transient monocyte depletion, but the factors that regulate recovery of monocyte populations are not fully understood. In this study, we investigated whether the composition of the gastrointestinal microbiota is associated with the recovery of monocyte homeostasis after HCT.

Methods: We performed a single-center, prospective, pilot study of 18 recipients of either autologous or allogeneic HCT. Serial blood and stool samples were collected from each patient during their HCT hospitalization. Analysis of the gut microbiota was done using 16S rRNA gene sequencing, and flow cytometric analysis was used to characterize the phenotypic composition of monocyte populations.
\end{abstract}

Results: Dynamic fluctuations in monocyte reconstitution occurred after HCT, and large differences were observed in monocyte frequency among patients over time. Recovery of absolute monocyte counts and subsets showed significant variability across the heterogeneous transplant types and conditioning intensities; no relationship to the microbiota composition was observed in this small cohort.

Conclusion: In this pilot study, a relationship between the microbiota composition and monocyte homeostasis could not be firmly established. However, we identify multivariate associations between clinical factors and monocyte reconstitution postHCT. Our findings encourage further longitudinal surveillance of the intestinal microbiome and its link to immune reconstitution.

(c) 2020 International Academy for Clinical Hematology. Publishing services by Atlantis Press International B.V. This is an open access article distributed under the CC BY-NC 4.0 license (http://creativecommons.org/licenses/by-nc/4.0/).

\section{INTRODUCTION}

Hematopoietic cell transplantation (HCT) is a potentially curative procedure for patients with hematologic malignancies, but its success has been limited by the morbidity and mortality of post-transplant infections and relapse. Impaired immune reconstitution post-HCT increases the risk of both complications [1-4]. Multiple factors influence the vulnerability of patients to these complications, including time since transplantation, graft source [i.e., autologous (auto-HCT) or allogeneic (allo-HCT)], and persisting myelosuppression (humoral and cell-mediated) post-HCT $[2,3]$. The composition of the intestinal microbiome is associated with clinical outcomes after allo-HCT, but the mechanisms for such have not been fully elucidated $[5,6]$.

“Corresponding authors.Email: morjaris@mskcc.org; michael.abt@pennmedicine.upenn.edu ${ }^{\dagger} \mathrm{MP}$ and MA are senior co-authors.

Peer review under responsibility of the International Academy for Clinical Hematology Data availability statement: The datasets generated during and/or analyzed during the current study are available from the corresponding author on reasonable request.
In humans, there are three main circulating monocyte subsets with diverse functions, classified based on their expression of CD14 and CD16 surface proteins and cytokine production. These monocyte subsets are named, "classical", "intermediate" and "nonclassical monocytes" [7-9], and typically comprise $85 \%, 10 \%$, and $5 \%$ respectively of the circulating monocyte pool in a healthy individual under homeostatic conditions $[10,11]$.

Classical monocytes specialize in phagocytosis and produce the cytokine interleukin (IL)-10 [12], while intermediate monocytes have elevated surface expression of MHC class II, suggesting they have an important role in antigen presentation [13]. In contrast, non-classical monocytes secrete substantial levels of inflammatory cytokines tumor necrosis factor (TNF) and (IL-1 $\beta$ ) and are known to exert endothelial surveillance by endovascular slow patrolling when tissue damage is present [14]. These roles make monocytes a critical cell line for host defense against common post-HCT infections including Aspergillus, and for mitigating the risk of developing graft-versus-host disease (GVHD) [15-21]. 
While it has been shown that monocyte reconstitution in the first 100 days post-HCT is associated with improved survival [22,23], a better understanding of the mechanisms that regulate monocyte reconstitution in this setting is needed. Given the known immunomodulating properties of the microbiota [24-28], we investigated whether the composition of the intestinal microbiota is associated with monocyte recovery. Monocyte migration out of the bone marrow and into the blood circulation has been shown to be driven by low level lipopolysacharide (LPS)-mediated signaling [29]. Further, basal circulating LPS levels derived from the intestinal microbiota have been detected in immunocompromised hosts [30,31]. Therefore, we hypothesized that microbial communities of Gram-negative bacteria through the production of LPS [32,33] and commensal anaerobes [34] influence monocyte maturation post-HCT.

\section{MATERIALS AND METHODS}

\subsection{Study Patients, Specimen Collection, and Patient Tracking}

The study cohort consisted of 18 adult recipients of auto- or alloHCT at the Memorial Sloan Kettering Cancer Center (MSKCC) from July 2015 to January 2016, as previously described [35].The gender breakdown consisted of seven females and 11 males with an age range from 40 to 75 . Longitudinal stool samples were collected during each patient's transplant hospitalization using a prospective institutional fecal biospecimen collection protocol (described previously) [36]. Daily stool collection began 7-10 days before hematopoietic cell infusion at the start of pre-transplant conditioning regimen and continued until discharge. The patients' median length of stay in the hospital was 27 days. Clinical metadata collected for all patients included medication administrations such as chemotherapy agents, antibiotics, absolute white blood cell values (determined by daily complete blood counts), and other patient characteristics that were retrieved from the electronic health record. The study protocol was approved by the Institutional Review Board (IRB \#15-124). Informed consent was obtained from all subjects prior to specimen collection.

\subsection{Transplantation Practices}

As previously described [35], antimicrobial prophylaxis was given routinely to patients undergoing HCT. Auto- or allo-HCT patients were given ciprofloxacin for 2 days prior to hematopoietic cell infusion as prophylaxis against Gram-negative bacterial infections. Allo-HCT recipients received intravenous (IV) vancomycin prophylactically against viridans-group streptococci [27] and either trimethoprim-sulfamethoxazole, aerosolized pentamidine, or atovaquone prophylaxis against Pneumocystis jiroveci pneumonia. The start of antibiotic prophylaxis administration during preconditioning or after HCT engraftment (defined as an absolute neutrophil count $\geq 500$ neutrophils $/ \mathrm{mm}^{3}$ for three consecutive days) varied. In the event of a new fever during times of neutropenia, patients were usually started on empiric antibiotics, such as piperacillin-tazobactam, cefepime, or meropenem. Recipients of an autograft received pegfiltastrim on day +1 and recipients of an allograft received daily filgastrim starting on day +7 until engraftment to accelerate recovery from neutropenia [37].

\subsection{Sample Analysis and Defining Microbial Predictors}

\subsubsection{Sample analysis}

Stool DNA was extracted and purified, and the V4 and V5 region of the $16 \mathrm{~S}$ rRNA gene was amplified by polymerase chain reaction using modified universal bacterial primers [38]. Sequencing was performed using the Illumina Miseq platform [39] to obtain paired-end reads. These reads were assembled, processed, filtered for quality, and grouped into operational taxonomic units of $97 \%$ similarity using a previously described UPARSE pipeline [24]. Taxonomic assignment to species level was performed using nucleotide Basic Local Alignment Search Tool [40] with the National Center for Biotechnology Information RefSeq (refseq_rna) as the reference database [41]. Alpha diversity was calculated using the inverse Simpson index at the OTU level [42] (For additional experimental details and microbiota data availability, see Supplementary Methods.).

\subsubsection{Microbial predictors}

We analyzed obligate anaerobic bacteria by major anaerobic groups defined at various taxonomic levels for their importance in maintaining 'healthy' immunity [34,43-46]: Clostridia (class), Bacterioidetes (phylum), Negativicutes (class) and Fusobacteria (genus). Percent anaerobes in a given stool sample was calculated by adding the percent $16 \mathrm{~S}$ rRNA gene sequences of these obligate anaerobic bacteria.

\subsection{Monocyte Subsets, Analysis of Blood Samples, Monocyte Isolation and Flow Cytometry}

A median of seven blood samples were obtained from each patient during transplant days -10 to +30 . A total of $5-10 \mathrm{cc}$ of blood in heparinized tubes were processed within $4 \mathrm{~h}$ of collection. The first blood sample was collected within 2 days of hospital admission (prior to any perturbations to white blood cells from chemotherapy or radiation) and subsequent blood samples were collected at the start of white blood cell reconstitution through engraftment $[47,48]$.

Peripheral blood mononuclear cells were isolated by density gradient centrifugation (Histopaque 1119; Sigma). Single-cell suspensions were stained for surface antigens with fluorescently conjugated antibodies and signals were acquired with LSR II (Becton Dickinson, Franklin Lakes, NJ). All flow cytometry data were analyzed using FlowJo software. For flow cytometry staining, the following antibodies were used: CD14-PE (clone M5E2; BD Biosciences, San Jose, CA), CD16-FITC (clone 3G8; BD Biosciences), CCR2-APC (clone K036C2; BioLegend, San Diego, CA), CD45-Alexa Fluor 700 (clone Hl30; BioLegend), CD11b (APC-Cy7; clone lCRF44; BioLegend), HLA-DR-PE-Texas Red (clone L243; BioLegend), CD86-PE-Cy7 (clone 1T2.2; BioLegend), CD15-Pacific Blue (clone H198; eBioscience), CD20-PerCP-Cy5.5 (clone 2H7; BioLegend), CD3-PerCP-Cy5.5 (clone OKT3; BioLegend), CD19-PerCP-Cy5.5 (clone SJ25C1; BioLegend), CD56-PerCP-Cy5.5 (clone 5.1H11; 
BioLegend), and CD5-PerCP-Cy5.5 (clone UCHT2; BioLegend). 'Fluorescent minus one' controls were used to determine positive staining gate [49]. The monocyte gating strategy used to define monocyte subsets (classical, intermediate, and non-classical) $[10,11,50]$ is shown with CD14 on the $x$-axis and CD16 on the $y$-axis. A dump gate excluded $\mathrm{B}$ cells, $\mathrm{T}$ cells, and NK cells using CD19/CD20, CD3, and CD56, respectively (Figure S2).

\subsection{Analytical Approach}

We analyzed absolute monocyte count recovery as a function of time at the start of immune reconstitution defined as engraftment day -2 days ("reconstitution day") until hospital discharge, using conditioning intensity and transplant type as stratification variables. Linear mixed models were fit using patient as a random effect (for intercept), absolute monocyte count as the dependent variable, and reconstitution day as a fixed effect. Fixed effect sizes and 95\% confidence intervals are shown.

Descriptive statistics (box plots) were used to visualize the proportions of classical (CD $\left.14^{\text {hi }} \mathrm{CD} 16^{\text {neg }}\right)$, intermediate $\left(\mathrm{CD} 14^{\mathrm{hi}} \mathrm{CD} 16^{\mathrm{Int}}\right)$, and non-classical (CD14 ${ }^{\text {Int }} \mathrm{CD} 16^{\text {hi }}$ ) monocytes in the last blood sample collected from each patient, to assess whether the subpopulations of circulating monocytes reached their estimated targets: approximately $85 \%$ classical monocytes, $10 \%$ non-classical monocytes and 5\% intermediate monocytes. These thresholds were determined based on previous studies $[10,11,51]$.

We calculated the Pearson correlation coefficient to measure the strength of the relationship between the proportion of anaerobic commensal gut microbes and microbial diversity to monocyte subset recovery. Next, we trained linear regression models while controlling for false discovery rates [52] to assess whether or not different clinical predictors correlated with monocyte recovery in the last blood sample tested (using the above mentioned 'target' values). The following clinical variables were used in the regression: (1) conditioning type [reduced-intensity conditioning (RIC) vs. myeloablative conditioning (MAC)] (2) transplant type (T-cell depleted vs. unmodified vs. auto-HCT) (3) GCSF administration within 7 days of blood collection (4) bloodstream infection (excluding positive blood cultures considered to be contaminants) within 7 days of blood collection and (5) percent Gram-negative bacteria (in the phyla, Bacteroidetes and Proteobacteria, major Gram-negative taxa) in the stool sample collected within 3 days before-or-after the last blood sample collected. Statistical analyses were performed using $\mathrm{R}$ (v. 3.3.1).

\section{RESULTS}

\subsection{Description of Study Population and Biospecimens}

Eighteen patients who received either an auto- or allo-HCT were included in this pilot study. They underwent different types of HCT for different hematologic malignancies. Four patients received an auto-HCT after MAC with Carmustine (BCNU), etoposide, cytarabine, and melphalan (BEAM). Four were given an allo-HCT after MAC consisting of the following regimens: total body irradiation, thiotepa, and cyclophosphamide or busulfan, melphalan, and fludarabine. Ten patients were recipients of an allo-HCT after RIC regimens. Clinical characteristics for each patient are shown in Table 1.

Patients stayed in the hospital for 20-38 days following their HCT and received both prophylactic and therapeutic antibiotics during this time. Throughout the hospitalization period, daily fecal sample collection was attempted resulting in a total of 236 samples that yielded high quality $16 \mathrm{~S}$ amplicons which could be sequenced. Concurrently, patient blood samples were collected for monocyte recovery analysis, with each blood sample being paired with a stool sample collected within 3 days (either before or after blood sample collection). A median of 15 stool samples and seven blood samples were collected per patient during their HCT hospitalization (Table 1).

\subsection{Monocyte Recovery}

Monocyte counts were considered during an analysis window from 2 days prior to the date of neutrophil engraftment ("reconstitution day") until hospital discharge. Significant differences in reconstitution trajectories were found among groups stratified by conditioning regimens and transplant type. Patients receiving reduced intensity conditioning and unmodified transplants demonstrated the most variability $\left(\mathrm{CI}_{95 \%}=[0.196 \pm 0.062]\right.$ and $\mathrm{CI}_{95 \%}=[0.194 \pm$ 0.060 ], respectively). All 18 patients reached a minimum normal absolute monocyte count value of $0.26 \times 10^{9} / \mathrm{L}$ defined elsewhere [53-55], and patients who received RIC and an unmodified transplant were more likely to overshoot the upper reference limit of $1.3 \times 10^{9}$ (a value determined by the MSKCC laboratory) (Figure $1 \mathrm{~A}$ and $1 \mathrm{~B})$.

Three representative flow cytometry plots collected longitudinally from three patients who received different transplant types [Auto-HCT; Allo-HCT (RIC); Allo-HCT (MAC)] show significant monocyte heterogeneity as represented by the commonly observed distribution of monocytes within a patient $[56,57]$ changing over time (Figure 2A). Data from all other patients $(n=15)$ are shown in Figure S1. The gating strategy used to identify the monocyte subsets of interest is shown in Figure S2.

Some patterns in monocyte reconstitution were observed; classical monocytes had an initial robust recovery consistent with previous findings [58], followed by restoration of intermediate and non-classical monocytes at later timepoints (Figure 2A). This trend was particularly pronounced for non-classical monocytes. On average, patients who were recipients of either an auto-HCT or allo-HCT with RIC were more likely than allo-HCT patients with MAC to recover classical monocytes to target threshold (approximately $85 \%)$. The threshold of approximately $5 \%$ intermediate monocytes was met in $72 \%(13 / 18)$ of patients, while those who received an allo-HCT with MAC were more likely to be discharged with levels of circulating non-classical monocytes that met target (approximately 10\%) (Figure 2B). Absolute levels of monocyte subsets were also calculated using the total white blood cell count $(/ \mu \mathrm{L})$ showing similar trends (Figure 2C).

We next compared the frequency of circulating monocyte subsets with the composition of the intestinal microbiota. At presentation, 
章

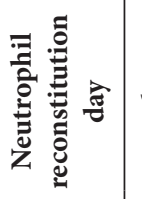

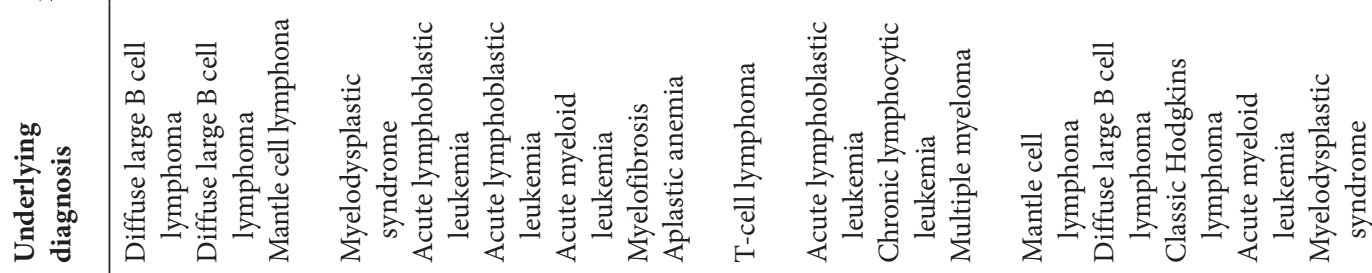

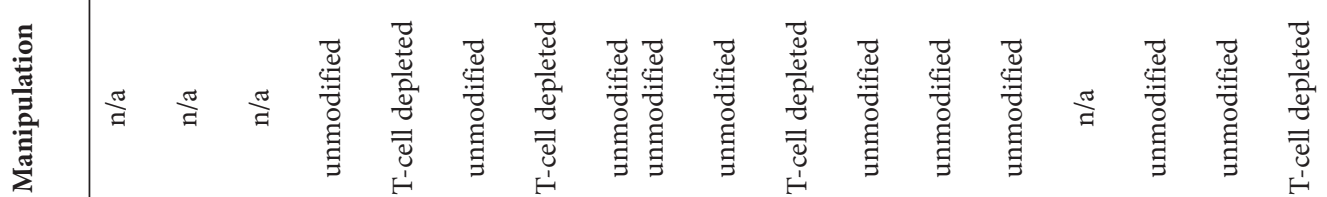

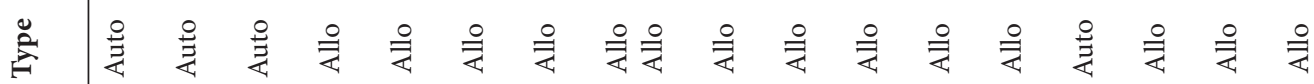

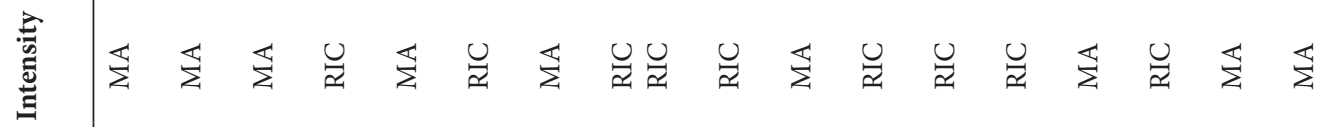

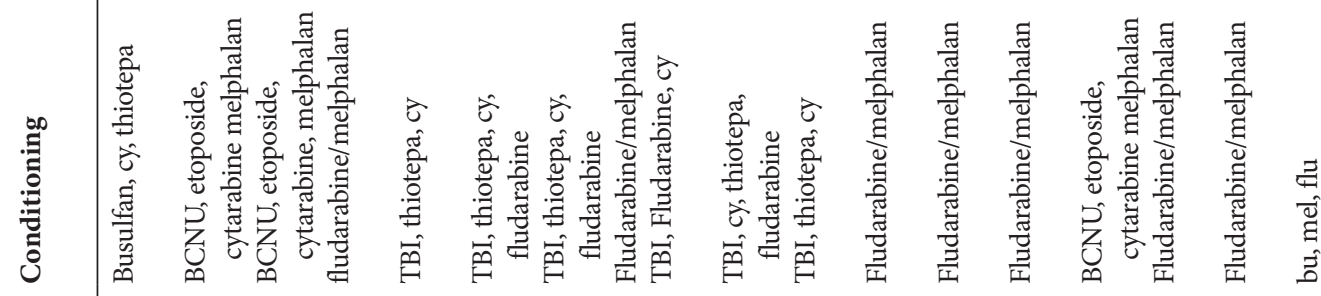



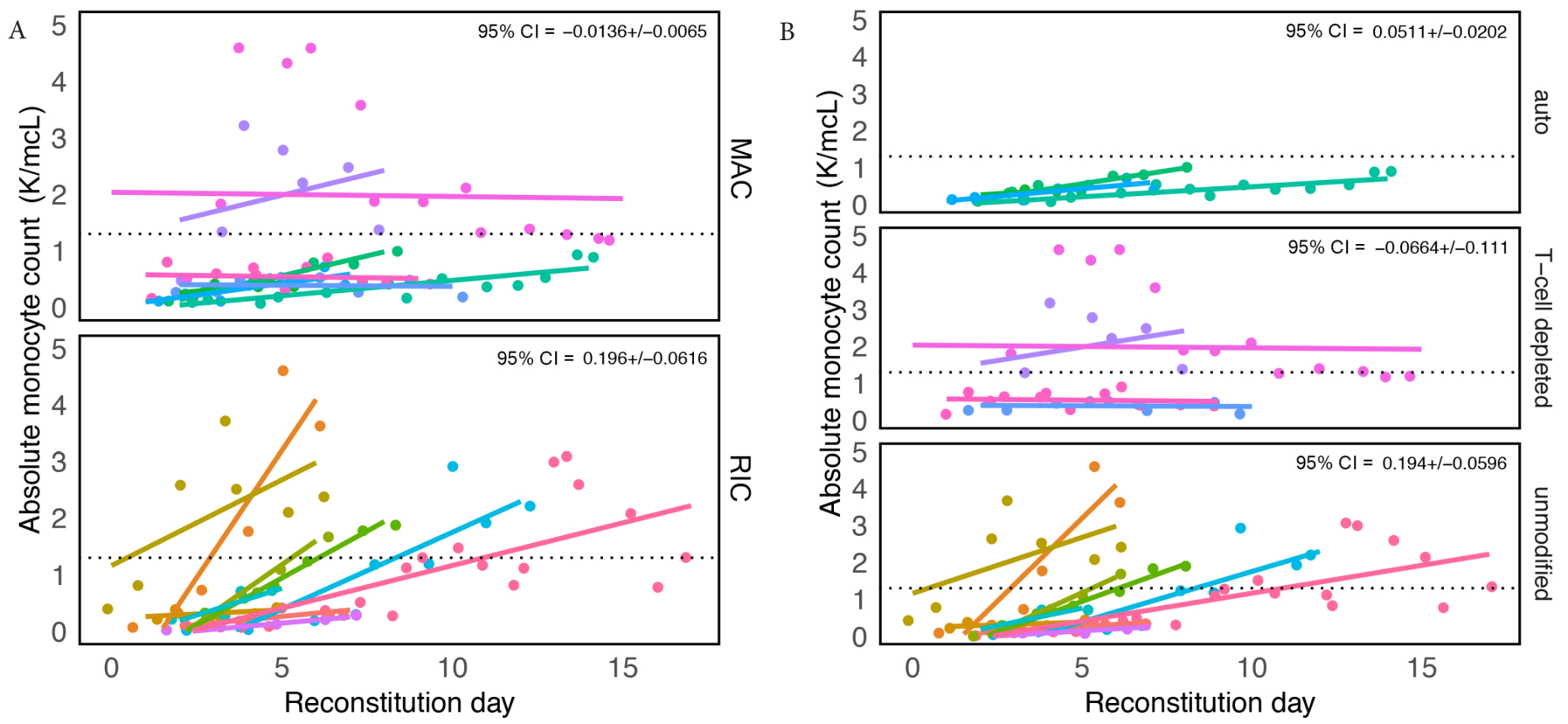

Figure 1 Recovery of absolute monocyte counts (AMC) is variable among patients receiving different conditioning regimens and transplant types. Graph showing absolute monocyte counts obtained for all patients when there was any sign of white blood cell recovery defined as "reconstitution day" (engraftment day - 2). Patient groups were divided by (A) conditioning, reduced intensity conditioning (RIC) and myeloablative conditioning (MAC) and (B) transplant type. Each color represents a different patient and a dashed line in each panel marks the upper limit of a "normal" AMC, defined by the Memorial Sloan Kettering Cancer Center laboratory to be $1.3 \mathrm{~K} / \mathrm{mcL}$. In the upper right-hand corner is the coefficient (i.e. slope/effect size) multiplied by 1.96 ( $\pm 95 \%$ confidence interval).

patients started with highly diverse intestinal microbial communities which subsequently underwent stark composition changes and loss of diversity upon antibiotic administration [35]. Diversity of intestinal microbial communities, as measured by inverse Simpson index, were not associated with monocyte recovery (Figure 3A). Additionally, the proportion of obligate anaerobes associated with a healthy flora (Negativacutes, Clostridia, Bacteroidiales, and Fusobacteria) within the microbiota did not correlate with monocyte recovery (Figure 3B).

While the proportion of monocyte subset recovery did not correlate with the microbiota, we next sought to investigate whether the immune activation status of monocyte subsets was altered by the microbiota composition. Monocyte immune activation phenotype as measured by the expression of cell surface markers [CD86, HLA-DR, and CCR-2 (Table S1)]. was assessed in each subset. No correlation was observed between expression of these markers and microbiota diversity (Figure S3) or percent of obligate anaerobic bacteria in the microbiota (Figure S4).

We used a simple linear regression model to test whether different compositional characteristics of the microbiota and different clinical variables are related to monocyte recovery. In this model, microbiota diversity and the proportion of Gramnegative Proteobacteria in the gut were not observed to be associated with monocyte recovery of each subset. Exposure to GCSF within 7 days of blood sample collection/processing was associated with successful reconstitution of classical and intermediate monocytes ( $p=0.037$ and 0.002 , respectively). Conversely, a T-cell depleted transplant was negatively associated with these monocyte subsets ( $p=0.041$ and 0.008 , respectively) Table 2 .
Non-classical monocytes were not significantly associated with any of the clinical predictors we defined.

\section{DISCUSSION}

Hematopoietic cell transplantation involves the administration of intense chemotherapy with or without radiation and antibiotic regimens, resulting in large shifts in leukocyte and microbiota compartments, often leading to complete compositional changes from 1 day to the next. The myeloablation associated with HCT provides a unique platform for exploring the nascent, re-development of immune reconstitution. Prior studies have supported the symbiotic relationship between the gut microbiota and the systemic immune system $[59,60]$, but most of that work has focused on the role the microbiota has on shaping different populations of T cells [29-31]. Staffas et al. [6] did find a delay in monocyte recovery when the gut flora was depleted with antibiotics, but it was not as dramatic as the defects in lymphocyte maturation post-HCT (in mice). We followed a systematic strategy to identify three subsets of monocytes (classical, intermediate, and non-classical monocytes) [10] during the period of immune reconstitution post-HCT to assess whether constituents of the microbiota affect monocyte recovery.

This pilot study involved high-frequency timeseries collection of stool and blood samples from 18 patients to assess whether a link between the microbiota and monocyte recovery could be found. We found no correlation between the microbiota composition and differences in monocyte recovery when analyzing a highdiversity microbiota or a microbiota composed primarily of either commensal anaerobes or Gram-negative organisms. We also found 

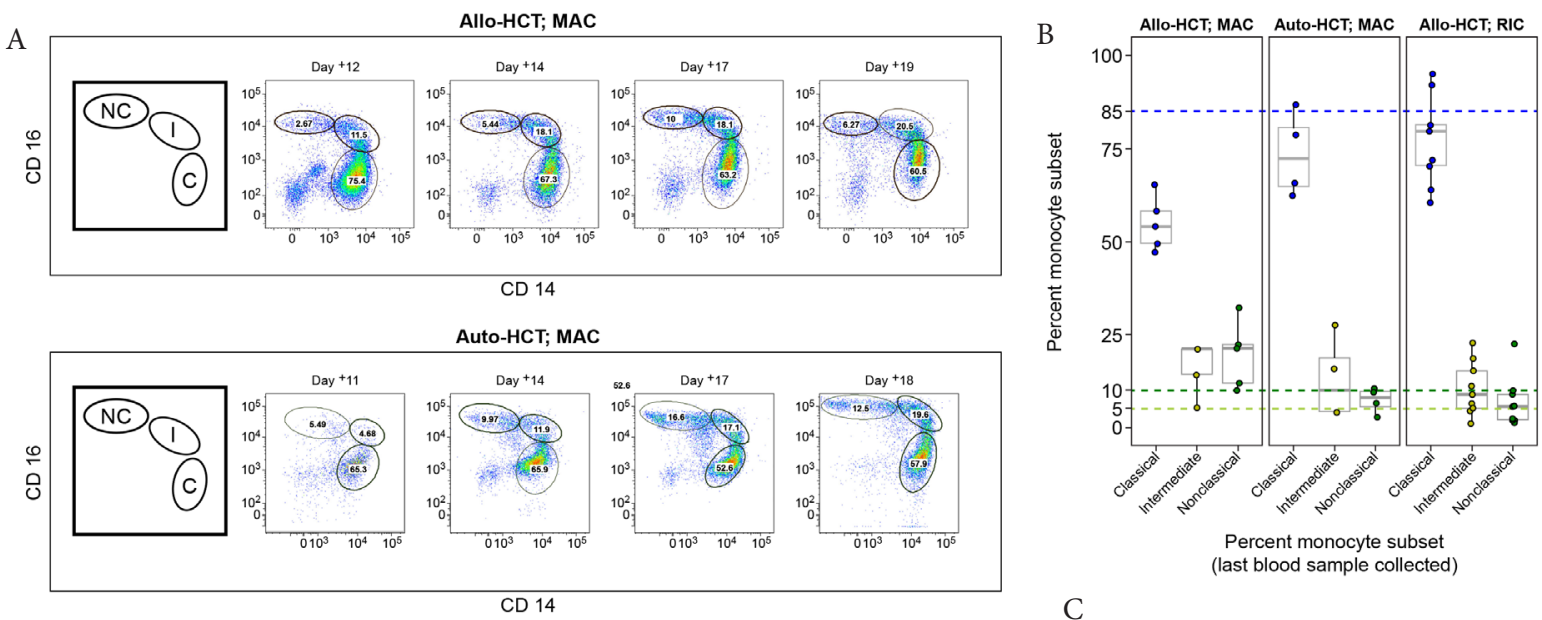

C
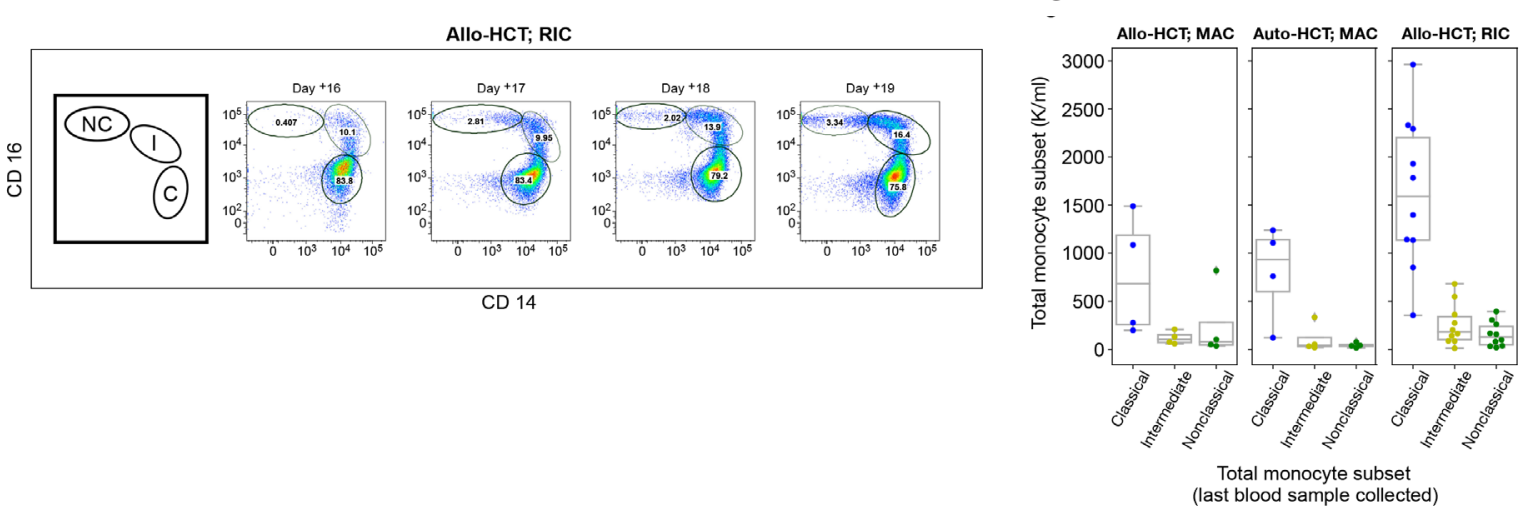

Figure 2 Variable recoveries of monocyte populations (classical, intermediate, and non-classical). (A) Time series for three representative patients using transplant day: Auto-hematopoietic cell transplantation (HCT) (top panel), Allo-HCT [reduced intensity conditioning (RIC; middle panel)], and Allo-HCT myeloablative conditioning (MAC; bottom panel). The first figure (from left-to-right for each panel) is a cartoon illustration depicting each monocyte subtype, classical (C), intermediate (I), and non-classical (NC) (clockwise from left-to-right) that corresponds to successive multicolor flow cytometry plots that follow. The numbers in each gate are percentages of each monocyte population. The gating strategy is detailed in Figure S1. (B) A boxplot showing the distribution of monocyte subsets determined from a patient's last blood sample collected. Colored dashed lines indicate the upper limit of "normal" for the percent values for each monocyte subset: $85 \%$ classical monocytes (blue), $10 \%$ non-classical monocytes (yellow), $5 \%$ intermediate monocytes (green). Data points (dots) indicate the monocyte subset type using the same color scheme. (C) A boxplot showing the distribution of absolute monocyte subsets from a patient's last blood sample and collected and calculated now using the patient's white blood cell count $(\mathrm{K} / \mathrm{mcL})$ taken from the electronic health record; the absolute value (classical, intermediate, non-classical) was determined by taking the white blood cell count $(\mathrm{K} / \mathrm{mcL}) \times 1000 \times$ percent cells in gates $(\mathrm{B}-\mathrm{G}$; Figure S2).
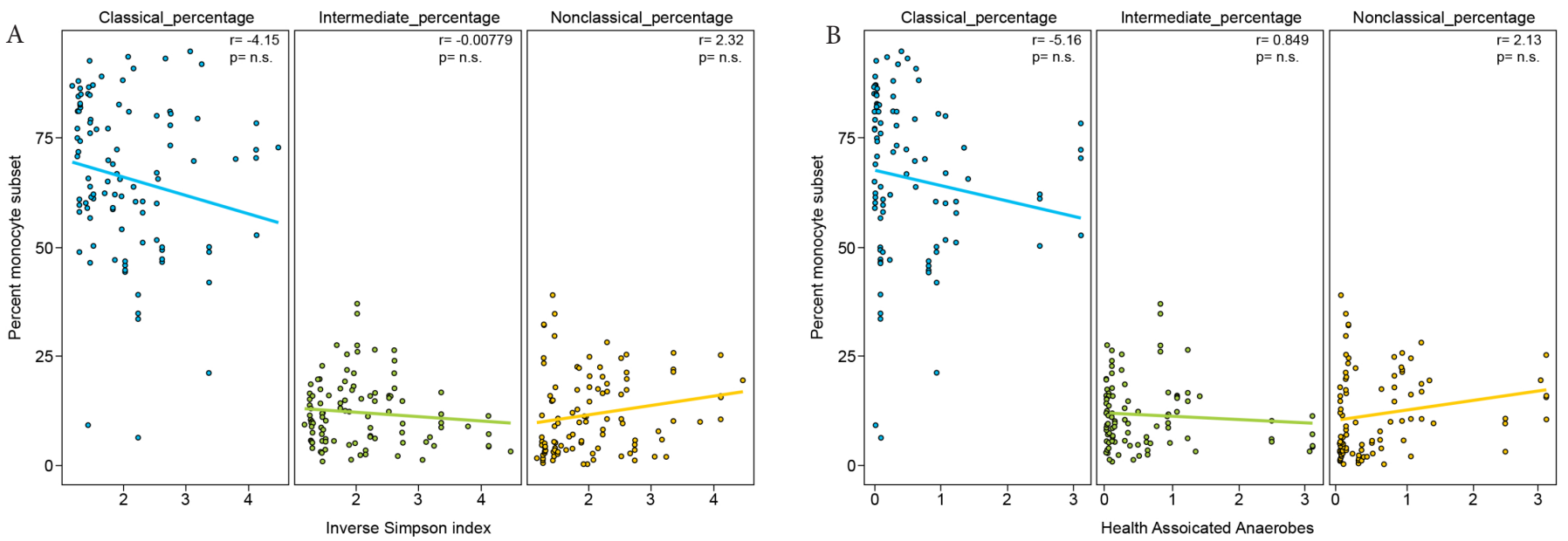

Figure 3 Monocyte subset frequency does not correlate with microbiota diversity or proportion of obligate anaerobes. (A) Relationship between the proportion of each monocyte subset and microbiota diversity measured by Inverse Simpson and (B) the proportion of obligate anaerobes (percent $16 \mathrm{~S}$ rRNA gene sequences of Negativacutes + Clostridia + Bacteroidiales + Fusobacteria) in the stool microbiota for each matched stool and blood collection. Pearson correlation coefficient values are shown in the upper right-hand corner of each panel. 
Table 2 Parameters assessed for association with monocyte subset recovery

\begin{tabular}{|c|c|c|c|}
\hline Variables & Type & Estimate & $p$-value Adj. \\
\hline$\%$ Gram-negatives in stool sample & Classical \% & -0.053 & 0.800 \\
\hline RIC & Classical \% & -0.88 & 0.540 \\
\hline Auto-HCT & Classical \% & 0.871 & 0.900 \\
\hline T-cell depleted & Classical \% & -1.103 & $0.041^{* *}$ \\
\hline Unmodified & Classical \% & -0.0792 & 0.106 \\
\hline+ Blood culture within 7 days of collection & Classical \% & 0.145 & 0.315 \\
\hline GCSF administration within 7 days of collection & Classical \% & 1.200 & $0.037^{* *}$ \\
\hline$\%$ Gram-negatives in stool sample & Intermediate \% & 0.146 & 0.458 \\
\hline RIC & Intermediate \% & -0.265 & 0.492 \\
\hline Auto-HCT & Intermediate \% & -1.395 & $0.003^{*}$ \\
\hline T-cell depleted & Intermediate \% & -1.310 & $0.008^{* *}$ \\
\hline Unmodified & Intermediate \% & 1.236 & $0.007^{* *}$ \\
\hline+ Blood culture within 7 days of collection & Intermediate \% & -0.033 & 0.931 \\
\hline GCSF administration within 7 days of collection & Intermediate \% & 1.401 & $0.002^{* *}$ \\
\hline$\%$ Gram-negatives & Non-classical \% & 0.039 & 0.892 \\
\hline RIC & Non-classical \% & 0.325 & 0.847 \\
\hline Auto-HCT & Non-classical \% & -0.293 & 0.847 \\
\hline T-cell depleted & Non-classical \% & 0.772 & 0.459 \\
\hline Unmodified & Non-classical \% & 0.244 & 0.846 \\
\hline + Blood culture within 7 days of collection & Non-classical \% & -0.893 & 0.254 \\
\hline GCSF administration within 7 days of collection & Non-classical \% & 0.895 & 0.318 \\
\hline
\end{tabular}

no association between microbiota composition and the expression of co-stimulatory markers, including CD86, HLA-DR and the chemokine receptor, CCR2.

Analysis of factors associated with immune reconstitution revealed that the relationship between the microbiota and immune reconstitution is weak relative to other clinical variables. As expected, GCSF exposure correlated positively with classical and intermediate monocyte reconstitution, while $\mathrm{T}$ cell depletion by means of CD34-positive selection was negatively associated with monocyte subset reconstitution. We also found significant variability in the reconstitution of absolute monocyte counts and monocyte subsets across patients. The biological significance of the varying rate of monocyte recovery on patient outcomes will need to be further explored.

Discerning the effects of many patient variables in the setting of HCT, including medications delivered and clinical complications that ensue (bacteremia, fever, GVHD, etc.) makes forming a direct relationship between the microbiota and monocytes in HCT patients challenging [61]. Medications given to HCT patients that have immunomodulating properties (i.e. antibiotics and steroids), could have served as confounders in our analysis. For example, $17 / 18$ patients received ciprofloxacin and 7/18 patients received steroids (not shown). Ciprofloxacin can dampen the effects of LPS $[62,63]$, a potent stimulator of monocyte mobilization out of the bone marrow [29,64], while steroids can reduce blood monocyte numbers $[29,65]$. Further, the intestinal permeability of LPS into systemic circulation to stimulate monocyte production from the bone marrow is not fully known, and testing for this endotoxin in serum or blood is challenging [61].

While we did not find an association between microbiota composition and monocyte recovery in this heterogeneous pilot cohort, this study demonstrates the feasibility of high-resolution sampling of blood and stool samples and opens the door to future studies where sampling more patients with daily frequency may help distinguish which microbial and clinical factors drive HCT patient outcomes. Future larger studies may reveal relationships between microbiota composition and immune reconstitution $[10,11]$.

\section{CONFLICTS OF INTEREST}

Dr. Perales reports honoraria from Abbvie, Bellicum, Bristol-Myers Squibb, Incyte, Merck, Novartis, Nektar Therapeutics, Omeros, and Takeda. He serves on DSMBs for Servier and Medigene, and the scientific advisory boards of MolMed and NexImmune. He has received research support for clinical trials from Incyte, Kite/Gilead and Miltenyi Biotec. He serves in a volunteer capacity as a member of the Board of Directors of American Society for Transplantation and Cellular Therapy (ASTCT) and Be The Match (National Marrow Donor Program, NMDP), as well as on the CIBMTR Cellular Immunotherapy Data Resource (CIDR) Committee. The other authors declare no conflict of interests.

\section{AUTHORS' CONTRIBUTION}

EP, MP and MA conceived of the presented idea. SM performed the laboratory experiments and constructed the paper. AZ and SK performed computational methods/data analysis. All authors discussed the results and contributed to the final manuscript.

\section{FUNDING}

This work was supported by the National Institutes of Health (grant R00-AI125786 to M.C.A.; U01Al124275-03 to E.G. P; R01CA228358 to M.v.d.B; P30 CA008748 MSKCC Support Grant/Core Grant, and Project 4 of P01-CA023766 to R. J. O’Reilly/M.v.d.B.). This work was further supported by the Parker Institute for Cancer 
Immunotherapy at Memorial Sloan Kettering Cancer Center; the Sawiris Foundation; the Society of Memorial Sloan Kettering Cancer Center; MSK Cancer Systems Immunology Pilot Grant, and Empire Clinical Research Investigator Program.

\section{SUPPLEMENTARY MATERIALS}

Supplementary data related to this article can be found at https:// doi.org/10.2991/chi.k.201108.002.

\section{REFERENCES}

[1] Sable CA, Donowitz GR. Infections in bone marrow transplant recipients. Clin Infect Dis 1994;18;273-81; quiz 282-4.

[2] Maury S, Mary JY, Rabian C, Schwarzinger M, Toubert A, Scieux $\mathrm{C}$, et al. Prolonged immune deficiency following allogeneic stem cell transplantation: risk factors and complications in adult patients. Br J Haematol 2001;115;630-41.

[3] Small TN, Papadopoulos EB, Boulad F, Black P, Castro-Malaspina $\mathrm{H}$, Childs BH, et al. Comparison of immune reconstitution after unrelated and related T-cell-depleted bone marrow transplantation: effect of patient age and donor leukocyte infusions. Blood 1999;93;467-80.

[4] Goldberg JD, Zheng J, Ratan R, Small TN, Lai KC, Boulad F, et al. Early recovery of T-cell function predicts improved survival after T-cell depleted allogeneic transplant. Leuk Lymphoma 2017;58;1859-71.

[5] Taur Y, Jenq RR, Perales MA, Littmann ER, Morjaria S, Ling L, et al. The effects of intestinal tract bacterial diversity on mortality following allogeneic hematopoietic stem cell transplantation. Blood 2014;124;1174-82.

[6] Staffas A, Burgos da Silva M, Slingerland AE, Lazrak A, Bare CJ, Holman CD, et al. Nutritional support from the intestinal microbiota improves hematopoietic reconstitution after bone marrow transplantation in mice. Cell Host Microbe 2018;23;447.e4-57.e4.

[7] Landmann R, Knopf HP, Link S, Sansano S, Schumann R, Zimmerli W. Human monocyte CD14 is upregulated by lipopolysaccharide. Infect Immun 1996;64;1762-9.

[8] Ziegler-Heitbrock L. Blood monocytes and their subsets: established features and open questions. Front Immunol 2015;6;423.

[9] Wong KL, Tai JJ, Wong WC, Han H, Sem X, Yeap WH, et al. Gene expression profiling reveals the defining features of the classical, intermediate, and nonclassical human monocyte subsets. Blood 2011;118;e16-e31.

[10] Marimuthu R, Francis H, Dervish S, Li SCH, Medbury H, Williams H. Characterization of human monocyte subsets by whole blood flow cytometry analysis. J Vis Exp 2018;140;57941.

[11] Ziegler-Heitbrock L, Ancuta P, Crowe S, Dalod M, Grau V, Hart $\mathrm{DN}$, et al. Nomenclature of monocytes and dendritic cells in blood. Blood 2010;116;e74-e80.

[12] Saha P, Geissmann F. Toward a functional characterization of blood monocytes. Immunol Cell Biol 2011;89;2-4.

[13] Hristov M, Weber C. Differential role of monocyte subsets in atherosclerosis. Thromb Haemost 2011;106;757-62.

[14] Belge KU, Dayyani F, Horelt A, Siedlar M, Frankenberger M, Frankenberger B, et al. The proinflammatory $\mathrm{CD} 14^{+} \mathrm{CD} 16^{+} \mathrm{DR}^{++}$ monocytes are a major source of TNF. J Immunol 2002;168;3536-42.
[15] Serbina NV, Cherny M, Shi C, Bleau SA, Collins NH, Young JW, et al. Distinct responses of human monocyte subsets to Aspergillus fumigatus conidia. J Immunol 2009;183;2678-87.

[16] Diamond RD, Huber E, Haudenschild CC. Mechanisms of destruction of Aspergillus fumigatus hyphae mediated by human monocytes. J Infect Dis 1983;147;474-83.

[17] Serbina NV, Shi C, Pamer EG. Monocyte-mediated immune defense against murine Listeria monocytogenes infection. Adv Immunol 2012;113;119-34.

[18] Smith MS, Bentz GL, Alexander JS, Yurochko AD. Human cytomegalovirus induces monocyte differentiation and migration as a strategy for dissemination and persistence. J Virol 2004;78;4444-53.

[19] Konuma T, Kohara C, Watanabe E, Mizukami M, Nagai E, Oiwa-Monna $\mathrm{M}$, et al. Circulating monocyte subsets in human chronic graft-versus-host disease. Bone Marrow Transplant 2018;53;1532-40.

[20] Chan G, Nogalski MT, Yurochko AD. Human cytomegalovirus stimulates monocyte-to-macrophage differentiation via the temporal regulation of caspase 3. J Virol 2012;86;10714-23.

[21] Rommeley M, Spies-Weisshart B, Schilling K, Hochhaus A, Sayer HG, Scholl S. Reconstitution and functional analyses of neutrophils and distinct subsets of monocytes after allogeneic stem cell transplantation. J Cancer Res Clin Oncol 2011;137;1293-300.

[22] Thoma MD, Huneke TJ, DeCook LJ, Johnson ND, Wiegand RA, Litzow MR, et al. Peripheral blood lymphocyte and monocyte recovery and survival in acute leukemia postmyeloablative allogeneic hematopoietic stem cell transplant. Biol Blood Marrow Transplant 2012;18;600-7.

[23] DeCook LJ, Thoma M, Huneke T, Johnson ND, Wiegand RA, Patnaik MM, et al. Impact of lymphocyte and monocyte recovery on the outcomes of allogeneic hematopoietic SCT with fludarabine and melphalan conditioning. Bone Marrow Transplant 2013;48;708-14.

[24] Chung H, Pamp SJ, Hill JA, Surana NK, Edelman SM, Troy EB, et al. Gut immune maturation depends on colonization with a host-specific microbiota. Cell 2012;149;1578-93.

[25] Smith K, McCoy KD, Macpherson AJ. Use of axenic animals in studying the adaptation of mammals to their commensal intestinal microbiota. Semin Immunol 2007;19;59-69.

[26] Belkaid Y, Hand TW. Role of the microbiota in immunity and inflammation. Cell 2014;157;121-41.

[27] Abt MC, Osborne LC, Monticelli LA, Doering TA, Alenghat T, Sonnenberg GF, et al. Commensal bacteria calibrate the activation threshold of innate antiviral immunity. Immunity 2012;37; $158-70$

[28] Maynard CL, Elson CO, Hatton RD, Weaver CT. Reciprocal interactions of the intestinal microbiota and immune system. Nature 2012;489;231-41.

[29] Shi C, Jia T, Mendez-Ferrer S, Hohl TM, Serbina NV, Lipuma L, et al. Bone marrow mesenchymal stem and progenitor cells induce monocyte emigration in response to circulating toll-like receptor ligands. Immunity 2011;34;590-601.

[30] Brenchley JM, Price DA, Schacker TW, Asher TE, Silvestri G, Rao S, et al. Microbial translocation is a cause of systemic immune activation in chronic HIV infection. Nat Med $2006 ; 12 ; 1365-71$.

[31] Di Lorenzo F, De Castro C, Silipo A, Molinaro A. Lipopolysaccharide structures of Gram-negative populations in the gut microbiota and effects on host interactions. FEMS Microbiol Rev 2019;43;257-72. 
[32] Munford RS. Sensing gram-negative bacterial lipopolysaccharides: a human disease determinant? Infect Immun 2008;76;454-65.

[33] Wang X, Quinn PJ. Endotoxins: lipopolysaccharides of gramnegative bacteria. Subcell Biochem 2010;53;3-25.

[34] Littman DR, Pamer EG. Role of the commensal microbiota in normal and pathogenic host immune responses. Cell Host Microbe 2011;10;311-23.

[35] Morjaria S, Schluter J, Taylor BP, Littmann ER, Carter RA, Fontana E, et al. Antibiotic-induced shifts in fecal microbiota density and composition during hematopoietic stem cell transplantation. Infect Immun 2019;87;e00206-19.

[36] Taur Y, Xavier JB, Lipuma L, Ubeda C, Goldberg J, Gobourne A, et al. Intestinal domination and the risk of bacteremia in patients undergoing allogeneic hematopoietic stem cell transplantation. Clin Infect Dis 2012;55;905-14.

[37] Gabrilove JL, Jakubowski A, Scher H, Sternberg C, Wong G, Grous J, et al. Effect of granulocyte colony-stimulating factor on neutropenia and associated morbidity due to chemotherapy for transitional-cell carcinoma of the urothelium. N Engl J Med 1988;318;1414-22.

[38] Chen K, Neimark H, Rumore P, Steinman CR. Broad range DNA probes for detecting and amplifying eubacterial nucleic acids. FEMS Microbiol Lett 1989;48;19-24.

[39] Caporaso JG, Lauber CL, Walters WA, Berg-Lyons D, Huntley J, Fierer N, et al. Ultra-high-throughput microbial community analysis on the Illumina HiSeq and MiSeq platforms. ISME J 2012;6;1621-4.

[40] Altschul SF, Gish W, Miller W, Myers EW, Lipman DJ. Basic local alignment search tool. J Mol Biol 1990;215;403-10.

[41] Tatusova T, Ciufo S, Fedorov B, O’Neill K, Tolstoy I. RefSeq microbial genomes database: new representation and annotation strategy. Nucleic Acids Res 2015;43;3872.

[42] Magurran AE. Measuring biological diversity. Malden, MA: Blackwell Publishing; 2004.

[43] Becattini S, Taur Y, Pamer EG. Antibiotic-induced changes in the intestinal microbiota and disease. Trends Mol Med 2016;22;458-78.

[44] Khosravi A, Yáñez A, Price JG, Chow A, Merad M, Goodridge HS, et al. Gut microbiota promote hematopoiesis to control bacterial infection. Cell Host Microbe 2014;15;374-81.

[45] Lee YJ, Arguello ES, Jenq RR, Littmann E, Kim GJ, Miller LC, et al. Protective factors in the intestinal microbiome against clostridium difficile infection in recipients of allogeneic hematopoietic stem cell transplantation. J Infect Dis 2017;215;1117-23.

[46] Jenq RR, Taur Y, Devlin SM, Ponce DM, Goldberg JD, Ahr KF, et al. Intestinal blautia is associated with reduced death from graft-versushost disease. Biol Blood Marrow Transplant 2015;21;1373-83.

[47] Wright DG, Meierovics AI, Foxley JM. Assessing the delivery of neutrophils to tissues in neutropenia. Blood 1986;67;1023-30.

[48] Ali MY, Oyama Y, Monreal J, Winter J, Tallman M, Gordon LI, et al. Reassessing the definition of myeloid engraftment after autotransplantation: it is not necessary to see $0.5 \times 10^{9} / 1$ neutrophils on 3 consecutive days to define myeloid recovery. Bone Marrow Transplant 2002;30;749-52.

[49] Passlick B, Flieger D, Ziegler-Heitbrock HW. Identification and characterization of a novel monocyte subpopulation in human peripheral blood. Blood 1989;74;2527-34.
[50] Ziegler-Heitbrock L. The $\mathrm{CD}_{14}{ }^{+} \mathrm{CD} 16^{+}$blood monocytes: their role in infection and inflammation. J Leukoc Biol 2007;81;584-92.

[51] Mukherjee R, Kanti Barman P, Kumar Thatoi P, Tripathy R, Kumar Das B, Ravindran B. Non-classical monocytes display inflammatory features: validation in sepsis and systemic lupus erythematous. Sci Rep 2015;5;13886.

[52] Benjamini Y, Hochberg Y. Controlling the false discovery rate: a practical and powerful approach to multiple testing. J R Stat Soc Ser B 1995;57;289-300.

[53] Adeli K, Raizman JE, Chen Y, Higgins V, Nieuwesteeg M, Abdelhaleem M, et al. Complex biological profile of hematologic markers across pediatric, adult, and geriatric ages: establishment of robust pediatric and adult reference intervals on the basis of the Canadian Health Measures Survey. Clin Chem 2015;61;1075-86.

[54] Horowitz GL, Altaie S, Boyd JC, Ceriotti F, Garg U, Horn P, et al. Defining, establishing, and verifying reference intervals in the clinical laboratory; approved guideline-third edition. Clinical and Laboratory Standards Institute; Vol. 28. 2010.

[55] Mayo Clinic Laboratories. Test ID: CBC. Complete Blood Count (CBC) with differential, blood. 2019. Available from: https://www. mayocliniclabs.com/test-catalog/Clinical+and+Interpretive/9109.

[56] Ziegler-Heitbrock HWL. Heterogeneity of human blood monocytes: the $\mathrm{CD} 14^{+} \mathrm{CD} 16^{+}$subpopulation. Immunol Today $1996 ; 17 ; 424-8$.

[57] Appleby LJ, Nausch N, Midzi N, Mduluza T, Allen JE, Mutapi F. Sources of heterogeneity in human monocyte subsets. Immunol Lett 2013;152;32-41.

[58] Patel AA, Zhang Y, Fullerton JN, Boelen L, Rongvaux A, Maini AA, et al. The fate and lifespan of human monocyte subsets in steady state and systemic inflammation. J Exp Med 2017;214;1913-23.

[59] Mazmanian SK, Liu CH, Tzianabos AO, Kasper DL. An immunomodulatory molecule of symbiotic bacteria directs maturation of the host immune system. Cell 2005;122;107-18.

[60] Gomez de Agüero M, Ganal-Vonarburg SC, Fuhrer T, Rupp S, Uchimura Y, Li H, et al. The maternal microbiota drives early postnatal innate immune development. Science 2016;351;1296-302.

[61] Hurley JC. Endotoxemia: methods of detection and clinical correlates. Clin Microbiol Rev 1995;8;268-92.

[62] Ziegeler S, Raddatz A, Hoff G, Buchinger H, Bauer I, Stockhausen A, et al. Antibiotics modulate the stimulated cytokine response to endotoxin in a human ex vivo, in vitro model. Acta Anaesthesiol Scand 2006;50;1103-10.

[63] Purswani MU, Eckert SJ, Arora HK, Noel GJ. Effect of ciprofloxacin on lethal and sublethal challenge with endotoxin and on early cytokine responses in a murine in vivo model. J Antimicrob Chemother 2002;50;51-8.

[64] Serbina NV, Pamer EG. Monocyte emigration from bone marrow during bacterial infection requires signals mediated by chemokine receptor CCR2. Nat Immunol 2006;7;311-17.

[65] Rinehart JJ, Sagone AL, Balcerzak SP, Ackerman GA, LoBuglio AF. Effects of corticosteroid therapy on human monocyte function. N Engl J Med 1975;292;236-41. 